\title{
A Hybrid Hyperquadric Model for 2-D and 3-D Data Fitting
}

\author{
Isaac COHEN, Laurent D. COHEN
}

\section{$\mathbf{N}^{\circ} 2188$}

January 1994

PROGRAMME 4

Robotique,

image

et vision

\section{apport}

\section{de recherche}





\title{
A Hybrid Hyperquadric Model for 2-D and 3-D Data Fitting
}

\author{
Isaac COHEN, Laurent D. COHEN \\ Programme 4 - Robotique, image et vision \\ Projet MASDA
}

Rapport de recherche $\mathrm{n}^{\circ} 2188$ - January 1994 - 34 pages

\begin{abstract}
We present in this paper a new curve and surface implicit model. This implicit model based on hyperquadrics allows a local and global control of the shape and a wide variety of allowable shapes. We define a hybrid hyperquadric model by introducing implicitly some local properties on a global shape model. The advantage of our model is that it describes global and local properties through a unique implicit equation yielding a representation of the shape by means of its parameters, independently of the chosen numerical resolution. The data fitting is obtained through the minimization of an energy, modelling the attraction to data independently of the implicit description of the shape.

After studying the geometry of hyperquadrics and how the shape deforms when we modify slightly its implicit equation, we are able to define an algorithm for automatic refining of the fit by adding an adequate term to the implicit representation. This geometric approach allows an efficient description of the data points and an automatic tuning of the fit according to the desired accuracy.
\end{abstract}

Key-words: Implicit curves and surfaces, Parametric models, Deformable models, Attraction potential, Segmentation, Surface reconstruction.

(Résumé : tsvp) 


\section{Approximation de données 2-D et 3-D à l'aide d'hyperquadriques hybrides}

Résumé : Nous présentons dans ce rapport un nouveau modèle de courbes et surfaces implicites. Ce modèle est basé sur les hyperquadriques permet un contrôle local et global de la forme et modélise une grande variété de formes. Ce modèle d'hyperquadriques hybrides est défini en introduisant d'une manière implicite quelques propriétés locales dans une représentation globale. Un des avantages de ce modèle consiste en la représentation de propriétés locales et globales par une équation implicite permettant de caractériser la forme de l'objet par l'intermédiaire des paramètres du modèle independemment de la résolution numérique. L'approximation des données est obtennue par la minimisation d'une énergie modélisant une force d'attraction independement de la description implicite de la forme.

Aprés avoir examiné les propriétés géométriques des hyperquadriques et l'évolution de la forme par rapport aux paramètres du modèle, nous avons défini un algorithme permettant d'améliorer l'approximation des données en rajoutant des termes à la représentation implicite. Cette approche géométrique permet une description fiable de données et un contrôle du nombre de paramètres en fonction de la précision souhaitée.

Mots-clé : Courbes et surfaces implicites, Modèles paramétriques, Modèles Déformables, Potentiels d'attraction, Segmentation, Reconstruction de surfaces 


\section{Introduction}

Using a shape representation in computer vision became a natural approach to describe and characterize a set of 2-D or 3-D data. Among the different approaches described in the computer vision research, we can classify these approaches in two distinct classes: free form models and parametric models. The first one refers to the spline model defined through its local degree of freedom allowing the modelling and the representation of a wide variety of shapes. In the spline model, the local properties stem from the local definition of the shape. This allows the reconstruction of a wide variety of surfaces represented by dense data. In the past years several authors $[5,6,10,18]$ have illustrated the use of free form model in image processing for modelling and characterizing image structures. On the other hand, the parametric model are defined in a global manner i.e. we have a unique equation chracterizing the surface. The different types of equations (algebraic,polynomial...) allow to characterize the shape through a small set of parameters. Several models were used: Algebraic surfaces of high degree [15],planar, quadratic, superquadric $[1,12,14]$ and also hyperquadric $[9,8]$ patches. The global definition of these models has proved it usefulness for defining a global constraint on the shape and for fitting very sparse data. Nevertheless, the choice of a model depends on the application and on the type of data the model have to deal with.

In this paper we address the problem of defining local property on a global shape model. More precisly, we establish how to define implicitly local properties in a global shape model. Several attempts were made to allow global and local shape control. In [17] the authors merge a global and a local model by defining a displacement field from a superquadric shape to fit 2-D and 3-D data. This displacement measuring the disparity between a superquadric and the fitted data, is not described through a spline model and cannot be represented through a small set of parameters.

Defining implicitly local properties on a global shape model yields a shape model defined through a unique set of parameters independently on the sparseness of the data. This allows to describe global and local properties through a unique implicit equation yielding a representation of the shape by means of its parameters.

RR $\mathrm{n}^{\circ} 2188$ 
In this paper we define a hybrid (global/local) geometric representation of implicit curves and surfaces. This representation allows us to define global and local constraints on the recovered shapes through a single parametric model. This model is based on the the hyperquadric model introduced by Hanson [9]. Recently, Han et al [8] have used an hyperquadric model for shape recovery. But the hyperquadric model does not allow the definition of local properties and remains a global convex shape model. On the contrary the hybrid hyperquadric model allows a local control of the shape, and the recovery of non-convex smooth shapes. The shape recovery from image data is done through a numerical parameterization of the recovered surface and the minimization of an energy, modelling the attraction to data independently of the implicit description of the shape. After studying the geometry of hyperquadrics and how their shape deforms when we modify slightly its implicit equation, we are able to define an algorithm for automatic refining of the fit by adding an adequate term to the implicit representation. This geometric approach allows an efficient description of the data points and an automatic tuning of the fit according to the desired accuracy.

This new approach allows to consider very complex implicit surface description of arbitrary degree. It represents an alternative to some approaches based on the elimination theory $[16,11]$ dedicated to polynomials of integer degree. The numerical parameterization enables also the use of the classic deformation primitives such as tapering, axial twist and bending [2]. Although some of these deformed shapes can be obtained through the proposed hybrid model, these primitives can be inserted directly in the numerical parameterization of the hybrid model to complete a wide range of shape modelling.

The work reported in this paper represents three major contributions.

- We define a new geometric representation of implicit curves and surfaces modelling a wide variety of shapes. The new model enhances the classical hyperquadric model since it allows an implicit definition of local properties like concavities.

- This new description is used for shape recovery from image data through a numerical parameterization of the recovered surface and the minimization of an energy, modelling the attraction to data independently of the implicit description of the shape. 
- We have defined an algorithm for automatic refining of the fit by adding an adequate term to the implicit representation. This geometric approach allows an efficient description of the data points and an automatic tuning of the fit according to the desired accuracy.

Finally we propose a new numerical parameterization allowing an efficient description of the shape characterized by the implicit equation.

The paper is organized as follows. Section 2 is on hyperquadrics model, focusing on the comparison with superquadrics, the definition of the hyperquadric model and on the use of a numerical parameterization. Section 3 explains the new hybrid hyperquadric model in detail. Section 4 is about fitting hybrid hyperquadric to data based on an energy minimization approach, it describes also a new geometric approach for an automatic refining of the fit by adding terms in the implicit representation. Section 5 is about fitting very sparse data and finally section 6 describes a new numerical parameterization allowing an efficient description of more general shapes. Illustrations and experimental results are given in the different sections to enlighten the model and the geometric methods used.

\section{The Hyperquadric Model}

In Computer Vision, most works using implicit surface models are based on superquadrics. These surfaces are included in a more general class of shape modelling, the hyperquadric model [9]. Before describing this model we recall first the definition of superquadrics. In the following we present the $3-\mathrm{D}$ surface models of superquadrics and hyperquadrics but naturally these models are defined and used similarly for 2-D curve modelling as showed in the examples and experimental results.

\subsection{Motivation}

The superquadrics are defined by the implicit equation:

$$
\left|\frac{x}{s_{1}}\right|^{\gamma_{1}}+\left|\frac{y}{s_{2}}\right|^{\gamma_{2}}+\left|\frac{z}{s_{3}}\right|^{\gamma_{3}}=1
$$

$\mathrm{RR} \mathrm{n}^{\circ} 2188$ 
or through the parameterization:

$$
\left\{\begin{array}{l}
x(\theta, \varphi)=s_{1} \operatorname{sign}(\cos \theta \cos \varphi)|\cos \theta \cos \varphi|^{\alpha_{1}} \\
y(\theta, \varphi)=s_{2} \operatorname{sign}(\sin \theta \cos \varphi)|\sin \theta \cos \varphi|^{\alpha_{2}} \\
z(\theta, \varphi)=s_{3} \operatorname{sign}(\sin \varphi)|\sin \varphi|^{\alpha_{3}}
\end{array}\right.
$$

where $\alpha_{i}=2 / \gamma_{i}, \theta \in[0,2 \pi[$ and $\varphi \in[-\pi / 2, \pi / 2]$.

These surfaces are fitted to the data through the minimization of different criteria depending on available data. All works reported on superquadric surfaces use the explicit parameterization of the surface. This parameterization is not available for hyperquadrics and induces a different approach for data fitting. An explicit parameterization of the shape has a major advantage: It alleviates the fitting process (whatever the chosen criterion is) since it gives an explicit control of the parameters defining the model, and also makes easier the use of deformation primitives [2].

Nevertheless, the limitations of superquadric models are due to the small set of allowable shapes, since the model is constrained by a few global parameters (such as radii, aspect ratios...) and consequently have a small number of degrees of freedom.

Our goal is to define implicitly some local properties on a global shape model. The advantage of such an approach is that the shape is still defined by a unique set of parameters independently of the chosen numerical resolution. The main point of this property is that the local control of the shape does not depend on the data resolution as in the case of deformable superquadrics [17] or spline models.

We propose a new method for defining implicitly local properties in a global shape model. This method applies to a wide variety of implicit function modelling (quadrics, superquadrics, algebraic surfaces). However, we choose a hyperquadric global shape model [9], since it includes most implicit models and have a wide variety of allowable shapes.

\subsection{Hyperquadrics}

The hyperquadrics are obtained by considering a sum of an arbitrary number of linear terms raised to powers [9]. This generates shapes whose bounding polytopes have arbitrary number of faces. 
A hyperquadric model is defined by the set of points satisfying

$$
H(x, y, z)=\sum_{i=1}^{N}\left|H_{i}(x, y, z)\right|^{\gamma_{i}}=1 .
$$

where $H_{i}$ is a $3-\mathrm{D}$ affine form:

$$
H_{i}(x, y, z)=\left(a_{i} x+b_{i} y+c_{i} z+d_{i}\right)
$$

where $a_{i}, b_{i}, c_{i}, d_{i}$ and $\gamma_{i}$ are constants $\left(\gamma_{i}>0\right)$.

Since $H(x, y, z)=1$ and all $\left|H_{i}\right|^{\gamma_{i}}$ are positive functions, we have for all $i$,

$$
\left|H_{i}(x, y, z)\right| \leq 1
$$

This last equation defines a strip bounded by the two hyperplanes $H_{i}(x, y, z)=1$ and $H_{i}(x, y, z)=-1$. The hyperquadric is thus included in the intersection of these strips which can be an arbitrary convex polytope (see figures 5 and 9 below).

If all the exponents $\gamma_{i}$ are greater than $1, H$ is a convex function and the inside set $\Omega$ defined by:

$$
\Omega=\left\{(x, y, z) \in \mathbb{R}^{3} \text { s.t. } H(x, y, z) \leq 1\right\}
$$

is convex. Alternatively, if some exponents are less than 1, we can recover non-convex shapes, although this introduces some singularities in the shape and makes the computation of the set of points $(x, y, z)$ solution of Eq. (3) very unstable.

Unlike the superquadrics, hyperquadrics do not have a general explicit parameterization because the number of terms $N$ exceeds in general the number of spherical variables. Hence the solutions of Eq. (3) are only obtainable through a numerical solution, which we details in the next section.

In Figure 1 we represent some 2-D hyperquadrics defined with three, four, and five terms, none of them is symmetric. This last feature clearly discriminates the hyperquadrics from the superquadrics model. Figure 2 shows some 3-D surfaces obtained with 5 and 6 terms, each line represents the same hyperquadric obtained with different exponents.

RR $\mathrm{n}^{\circ} 2188$ 


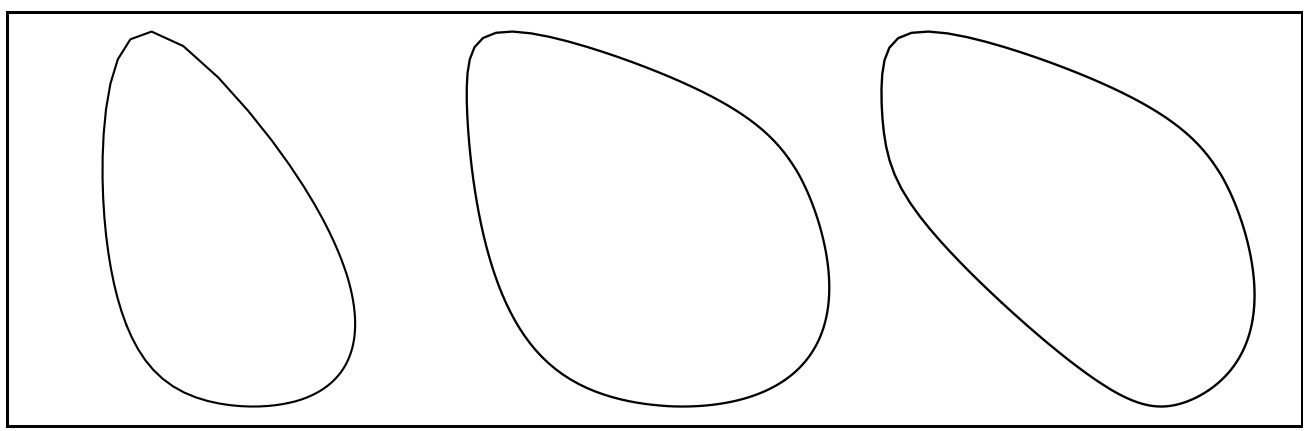

Figure 1: Some examples of 2-D hyperquadrics. The figure represents three hyperquadrics obtained with 3,4 and five terms with exponents greater than one.

\subsection{Hyperquadrics Numerical Representation}

An efficient approach to solve Eq. (3) numerically is to consider a radial parameterization of the hyperquadric:

$$
\left\{\begin{array}{l}
x(r, \theta, \varphi)=r(\theta, \varphi) \cos \theta \cos \varphi \\
y(r, \theta, \varphi)=r(\theta, \varphi) \sin \theta \cos \varphi \\
z(r, \theta, \varphi)=r(\theta, \varphi) \sin \varphi
\end{array}\right.
$$

where $r(\theta, \varphi) \geq 0, \theta \in[0,2 \pi]$ and $\varphi \in[-\pi / 2, \pi / 2]$. This parameterization is defined by searching for each $(\theta, \varphi)$ a scalar $r(\theta, \varphi)$ minimizing

$$
|H(X(r, \theta, \varphi))-1|^{2}
$$

where $X=(x, y, z)$. This minimization is done for each node $\left(\theta_{j}, \varphi_{k}\right)$ of a rectangular grid by using a Levenberg-Marquardt method [7].

Although the hyperquadric equation (3) can define some shape deformations like tapering, the numerical parameterization gives a much more common approach for shape deformations and a control of these deformations. Indeed one can easily deform a shape from its parameterization [2] and have an intuitive control of the deformations rather than to use some algebraic properties of the parameters defining the hyperquadric. These algebraic properties are not easy to obtain since we can have an arbitrary number of linear terms $H_{i}$ raised to arbitrary exponents. 


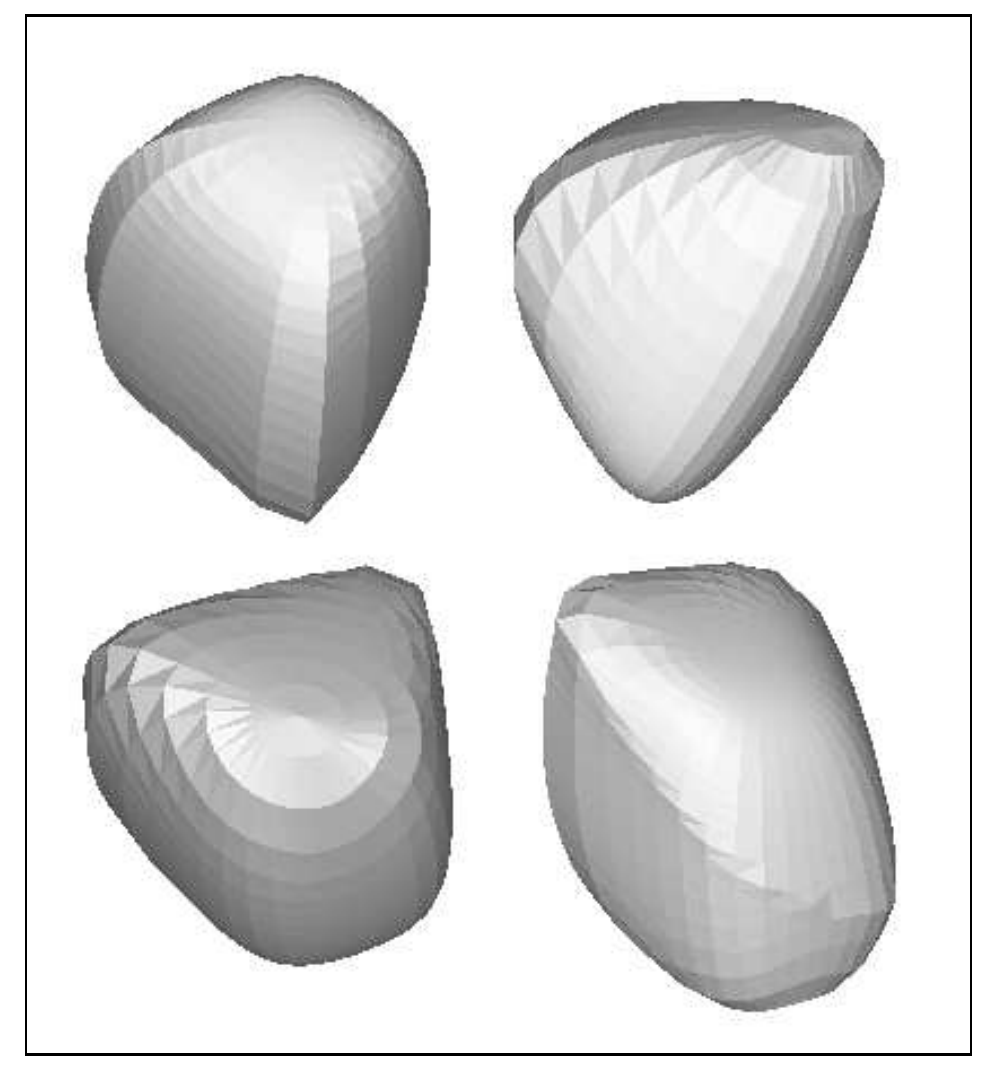

Figure 2: 3-D hyperquadrics obtained with 5 terms (top) and 6 terms (bottom). The surfaces on the right were obtained by choosing exponents lower than one for one 3-D linear form.

\section{The Hybrid Hyperquadric Model}

We now present our new method for defining implicitly local properties in a global shape model. This approach could also apply to a wide variety of implicit function modelling (quadrics, superquadrics, algebraic surfaces). However, we choose a hyperquadric global shape model [9], since it includes most implicit models and have a wide variety of allowable shapes.

In the hyperquadric model the shape is defined globally by the different linear forms. These forms define the convex bounding polytope which gives a geometric description of the shape. As we have seen this model allows to describe a wide variety of shapes since we can use an unlimited number of linear forms. However, the definition of non-convex shapes is still hard to achieve (numerical instability, appearance of cusps) and we cannot control 
sufficiently the shape and the location of the desired concavity. Furthermore the global shape is modified.

The main novelty of our approach is to incorporate in the hyperquadric model an arbitrary number of concavities or local proprieties at the desired locations.

Given some implicit equations representing different shapes, some authors have proposed to combine them to construct non-convex shapes. This was generally done by combining elliptic and hyperbolic shapes and computing the intersection of positive and negative volumes. Such surfaces have cusps at the intersection points and the obtained surfaces are not represented through a unique implicit equation. Furthermore, visualizing these surfaces usually produces artifacts. Instead we use the approach proposed by Hanson [9] based on combining exponential terms in the hyperquadric equation. This approach is similar to the negative volumes in the "blobby" model [3].

The shape produced by an exponential of hyperquadric is a "hill" centered at the center of the convex polytope of the hyperquadric. Adding these terms to a hyperquadric equation allows a local control of the shape and the location of these hills. In the following we combine these exponential shapes with hyperquadrics.

A hybrid hyperquadric or a composite hyperquadric is thus defined through an implicit equation of the type:

$$
H(x, y, z)=\sum_{i=1}^{N}\left|H_{i}(x, y, z)\right|^{\gamma_{i}}+\sum_{j=1}^{M} c_{j} \mathrm{e}^{-\sum_{l=0}^{L_{j}}\left|K_{j l}(x, y, z)\right|^{\gamma_{j l}}}=1
$$

where $H_{i}$ and $K_{j l}$ are 3-D linear forms like those defined in Eq. (4), $N$ represents the number of strips defining the hyperquadric, $M$ is the number of concavities used and $L_{j}$ 's are the numbers of strips defining the shape of each concavity. Remark that instead of using an exponential we could similarly use a function which has the same "bell" shape, like:

$$
\frac{1}{1+\sum_{l=0}^{L}\left|K_{l}(x, y, z)\right|^{\gamma_{l}}}
$$

for defining the concavities or the local properties of the shape.

Hence we end up with a parametric model defined by $5 \times\left(N+\sum_{j=1}^{M} L_{j}\right)$ parameters. These parameters define the global and the local shape of the hybrid hyperquadric. In the following, 


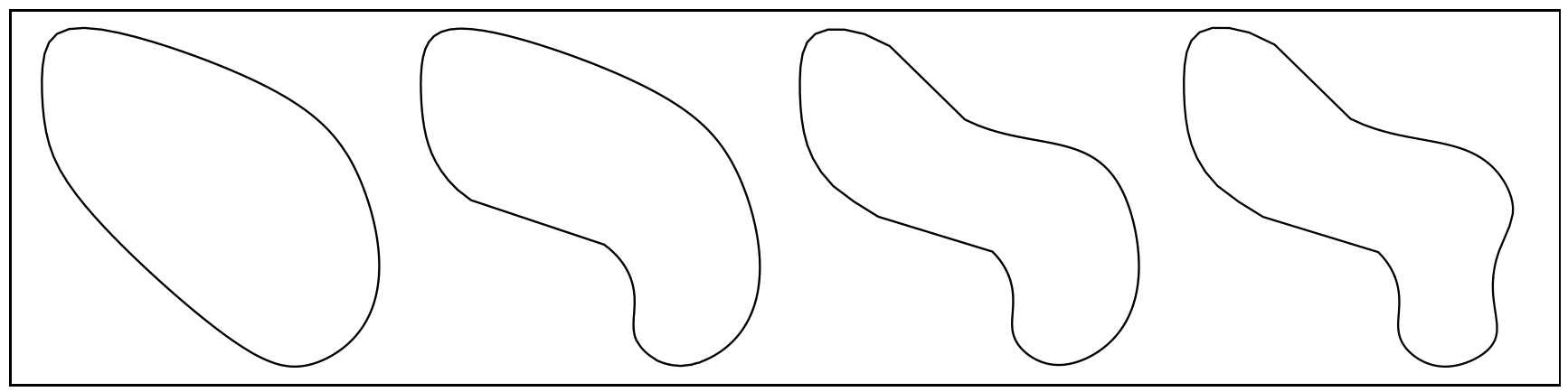

Figure 3: Inserting different types of exponential functions in a classical hyperquadric shape. This figure illustrates a hyperquadric defined with 5 terms (on the left) with one, two and three concavities added to the first shape. We can note that the definition of the concavities is local and do not deform the whole shape of the initial hyperquadric.

we represent the set of $5 \times\left(N+\sum_{j=1}^{M} L_{j}\right)$ parameters defining the hybrid hyperquadric by a vector $\mathcal{A}$.

Solution of the implicit equation (9) can be done in different ways. Hanson [9] have proposed to solve separately the hyperquadric part and the exponential part of the equation and then merge the surfaces patches. This yields a set of points satisfying Eq (9) without any global description of the whole shape i.e. we do not have a unique parameterization of the obtained surface.

To represent the shape of the hybrid hyperquadric, we use the numerical parameterization given in section 2.3. This radial parameterization constrains the set of allowable shape but it is an efficient representation of the surface. In section 6 we introduce a generalization of this approach allowing a parameterization of more complex hyperquadric shapes.

In figures 3 and 4 we show the use of hybrid hyperquadrics for $2-\mathrm{D}$ and $3-\mathrm{D}$ shape modelling. As showed, we can generate an arbitrary number of concavities and the definition of these concavities remains local and does not modify the global shape of the hyperquadric. With this hybrid model we have defined a parametric model which has global and local properties. The global shape properties are necessary if we wish to constrain the set of admissible shapes and the local properties of the model can be used to refine locally the 


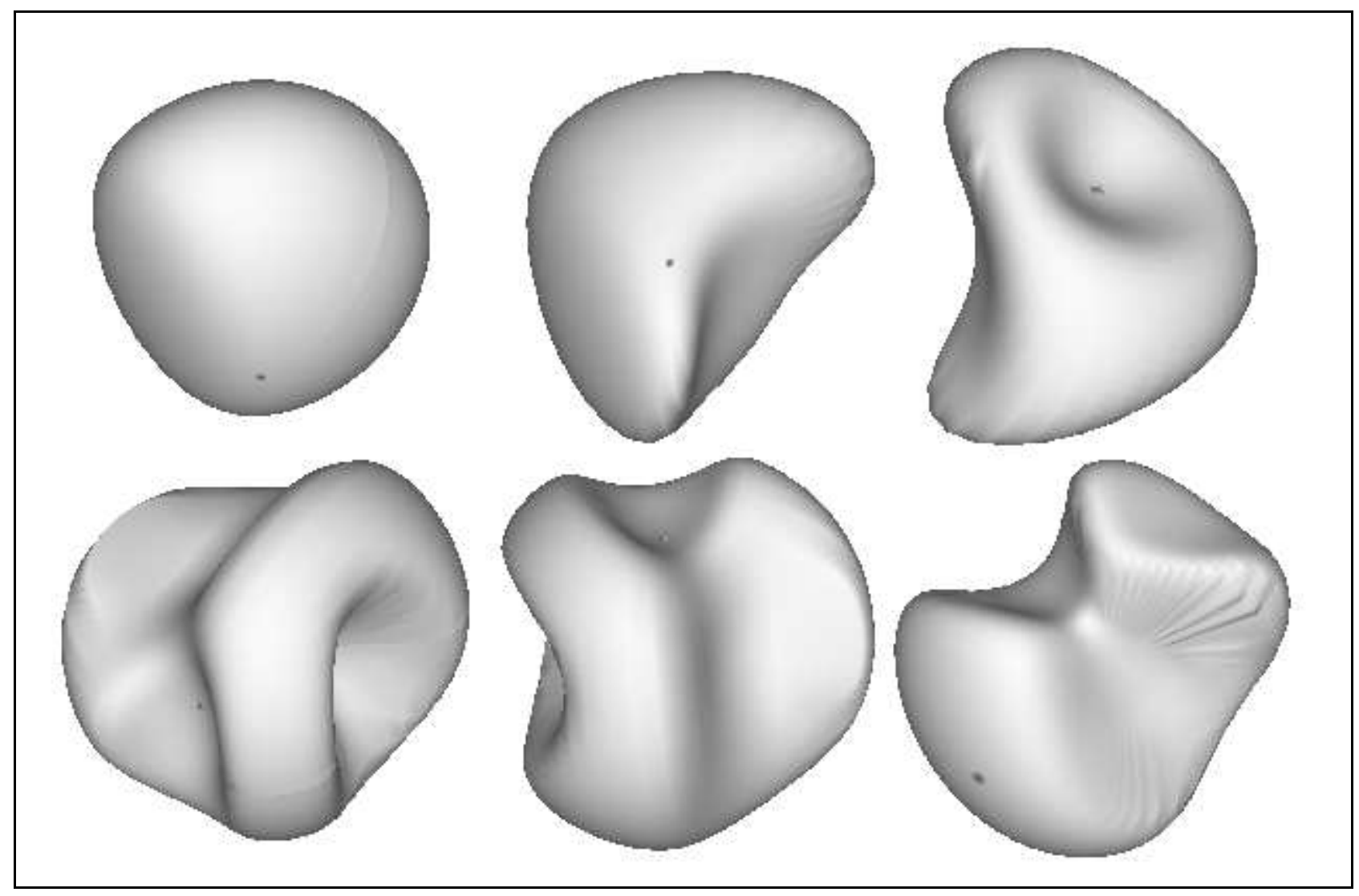

Figure 4: Definition of different types of concavities for shape modelling in 3-D surfaces. Here we show some 5 terms hyperquadric surfaces along with two and three exponential functions. In the first line we represent the initial hyperquadric and the successive addition of two concavities. This illustrates the wide variety of allowable hybrid hyperquadric shapes.

shape. The advantage of this approach is that the description of the shape remains an implicit equation defined by a small number of parameters. This new definition of local properties characterizes our model from the other deformable models $[6,18]$ or deformable superquadrics [17] allowing reconstruction of local properties through a large number of degrees of freedom of a spline. 


\section{$4 \quad$ Fitting Hybrid Hyperquadrics}

\subsection{Overview}

The fitting of a hyperquadric surface to 3 -D data points consists in recovering the set of parameters $\mathcal{A}$ defining the shape of the surface through the implicit Eq. (3). These parameters can be recovered in different ways. Two methods are usually used for fitting parametric models to data points:

- Inside-Outside functions: This method (see [14] for an example applied to superquadrics) is based on characterizing the implicit surface satisfying:

$$
H(x, y, z)=1
$$

where $H$ is defined in Eq. (9), as a boundary between points lying inside the hyperquadric and those lying outside the hyperquadric. Thus, for each 3 -D point $(x, y, z)$ we have the following classification:

$$
\left\{\begin{array}{l}
H(x, y, z)<1 \text { the point lies inside } \\
H(x, y, z)=1 \text { the point is on the boundary } \\
H(x, y, z)>1 \text { the point lies outside. }
\end{array}\right.
$$

Given a set of 3-D data points, one can use the above classification to fit the hyperquadric to the data by a least-squares minimization of the inside-outside function. The parameters recovery is done by minimizing:

$$
\sum_{d=0}^{D}\left|H\left(x_{d}, y_{d}, z_{d}\right)-1\right|^{2},
$$

where $D$ is the number of 3 -D data points. Several authors have noted that this criterion does not constrain enough the shape recovery of superquadric models $[14,4]$ since it does not measure efficiently the distance between the surface and the data.

- Euclidean distances: This method is based on minimizing the approximate distance between the surface and 3-D data points. The hyperquadric surface is defined through an arbitrary number of terms raised to arbitrary powers, thus the exact computation of

RR $n^{\circ} 2188$ 
the distance is computationally expensive and sometimes numerically unstable. Instead a first order approximation of the euclidean distance from a $3-\mathrm{D}$ point to a zero set of smooth functions is used $[15,8]$. Assume $X \in \mathbb{R}^{3}$ is a data point close to the hyperquadric surface and suppose $p$ is the closest point to $X$ on the surface (the point $p$ is not known explicitly) such that $H(p)=1$ and $\|\nabla H(p)\| \neq 0$, thus

$$
H(X)=1+\nabla H(p)(X-p)+\mathcal{O}\left(\|X-p\|^{2}\right)
$$

Hence a good approximation of the distance (see [15] for a complete discussion) near a regular point $p$ (i.e. such that $\|\nabla H(p)\| \neq 0$ ) is given by:

$$
\|X-p\| \simeq \frac{|H(X)-1|}{\| \nabla H(X) \mid}
$$

since the closest point is in the direction of $\nabla H(p) \simeq \nabla H(X)$. Fitting the hyperquadric to a set of $3-\mathrm{D}$ data points is done by a least-squares minimization problem:

Find the set of parameters $\mathcal{A}$ minimizing the distance between the hyperquadric surface and 3-D data points $X_{d}$ :

$$
\sum_{d=0}^{D}\left(\frac{\left|H\left(X_{d}\right)-1\right|}{\| \nabla H\left(X_{d}\right)||}\right)^{2}
$$

where $D$ is the number of 3-D data points.

Han et al [8] have used this criterion to recover hyperquadric shapes from image data. This approximate distance is biased, since if a data point $X_{d}$ is close to a critical point (i.e. a point $p$ such that $\|\nabla H(p)\| \simeq 0$ and $H(p) \neq 1$ ) the ratio given in Eq. (12) becomes large. This limitation constrains the set of admissible shape.

For the hyperquadrics, minimizing this approximate distance is safe only in recovering convex shapes. In this case, $\gamma_{i}>1, \forall i=1 . . N$, thus the polynomial $H$ given by Eq. (3), is strictly convex and $\|\nabla H(p)\| \neq 0$ except at its center point. This is no longer true for the hybrid hyperquadric model. With this model we can have critical points while having all the parameters $\gamma_{i}>1$ due to the exponential functions. An example is given by the hybrid hyperquadric curve:

$$
H(x, y)=\frac{x^{2}}{2}+\frac{y^{2}}{2}+\frac{1}{2} \exp \left(1-x^{2}-y^{2}\right)
$$


where we have $H(x, y)=1$ for each $(x, y)$ such that $x^{2}+y^{2}=1$, and over this set we also have $H^{\prime}(x, y)=0$. In this case the use of a criterion given by Eq. (12) could not provide a solution of $H(x, y)=1$. Hence the use of the criterion based on the approximate distance can give erroneous shapes in the case of hybrid hyperquadrics.

We have seen that the above methods are not appropriate for hybrid models, the insideoutside method does not perform well and the second approach could not be used with the exponential functions. In the following we propose a method based on an energy minimization method which characterizes the $3-\mathrm{D}$ data points we are trying to fit.

\subsection{Energy Minimizing Surface Approach}

The above criteria are relevant only if all data points are supposed to be part of the same object. When a segmentation task has to be done simultaneously to fit the model to part of the data, the previous approaches are not efficient.

An alternative approach is to use an energy based surface model. This physically based approach, used for deformable surfaces fitting $[5,18]$, constrains the set of admissible functions and defines a set of applied forces. This model can also be seen as a model of internal and external forces acting on the surface and the fitted surface is obtained through an equilibrium state of these forces. The internal forces are used to define the different degrees of freedom of the surface while the external forces characterize the data to be fitted.

In the hybrid hyperquadric model the set of admissible functions is already defined through the implicit equation (9) and consequently we do not need to use any internal force to constrain the set of admissible solutions. Henceforth the energy is defined only through the external forces (like in [19]). Let us consider a potential $P$ characterizing the external forces (i.e. the applied forces $\vec{f}$ are given by $\vec{f}=-\nabla P$ ). In the forthcoming we will choose a potential $P$ such that a hybrid hyperquadric will fit the data by minimizing the energy associated to the potential $P$ :

$$
E(\mathcal{A})=\int_{\mathcal{S}_{\mathcal{A}}} P(x, y, z) d x d y d z
$$

$\mathrm{RR} \quad \mathrm{n}^{\circ} 2188$ 
where $\mathcal{A}$ is the set of parameters defining the hyperquadric $H$ given by Eq. (9) and

$$
\mathcal{S}_{\mathcal{A}}=\left\{(x, y, z) \in \mathbb{R}^{3} \text { such that } H(x, y, z)=1\right\}
$$

defines the hyperquadric surface.

Fitting a hybrid hyperquadric to data is then equivalent to find the set of parameters $\mathcal{A}$ minimizing the functional $E(\mathcal{A})$ of Eq. (14).

\subsection{A Numerical Minimization Scheme}

The implicit description of the shape of a hybrid hyperquadric and the lack of an analytical explicit parameterization make the model much more difficult to handle. Indeed, we cannot express the functional $E$ in terms of the parameters defining the hyperquadric independently of the chosen potential $P$. Thus we cannot use a direct gradient based method since we cannot compute the derivatives:

$$
\frac{\partial E(\mathcal{A})}{\partial \mathcal{A}}=\frac{\partial}{\partial \mathcal{A}} \int_{\mathcal{S}_{\mathcal{A}}} P(x, y, z) d x d y d z
$$

This clearly differentiates the model from the superquadrics since the explicit parameterization given by Eq. 2 allows to compute numerically the derivatives, and consequently to use a gradient based method to recover the set of parameters defining the superquadric.

Instead we propose a two step numerical minimization of the energy $E$. This method is based on starting from an initial estimate of the shape,

1. deform it according to the applied forces $\vec{f}=-\nabla P$,

2. recover the hyperquadric parameters by minimizing the functional (11).

These two steps are iterated until we reach a minimum of the functional $E(\mathcal{A})(14)$.

This method differs from the classic ones since it separates the criterion of the goodness of fit and the recovery of the hybrid hyperquadric parameters. Although we use an insideoutside criterion (11) for the parameters recovery, we do not encounter the problem of how to estimate correctly the euclidean distance between the hyperquadric surface and the data points $[4,14,15]$ since it is done through the definition of the potential $P$ and is independent 
of the analytic equation of the surface. Also the data set to fit in step 2 is already segmented and all these data points are relevant for the parameters recovery. This approach is similar to the deformable surfaces model, since we deform the surface according to a potential and then recover the parameters of the hyperquadrics.

This process is equivalent to solve a partial differential equation describing the surface evolution during the fit. We can write it as:

$$
\left\{\begin{array}{l}
\frac{\partial \mathcal{A}}{\partial t}+\nabla_{\mathcal{A}} E(\mathcal{A})=0,(x, y, z) \in \mathcal{S}_{\mathcal{A}} \\
\mathcal{A}_{0} \text { Initial estimate (i.e initial shape). }
\end{array}\right.
$$

where $\mathcal{A}$ is the set of parameters defining the hybrid hyperquadric $H_{\mathcal{A}}(x, y, z)$, the parameter $\mathcal{A}_{0}$ defines the initial shape and $\nabla_{\mathcal{A}} E(\mathcal{A})$ represents the derivatives of the energy (Eq. 16) with respect to the parameter $\mathcal{A}$. A stationary solution of Eq. (17) represents the parameters of the surface fitted to the data points. As noted before, one could solve Eq. (17) if an analytical description of $\mathcal{S}_{\mathcal{A}}$ was available. This amounts to have an explicit parameterization of the hybrid hyperquadrics.

\subsection{Defining the Potential $P$}

Deforming a shape according to the minimization of a potential becomes a classical approach in image segmentation since the use of "Snakes" [10] and deformable surfaces [5, 18]. A number of authors $[5,6]$ (and references therein) have showed that the use of a potential for characterizing the data models a large set of attraction forces and allows to incorporate other extracted informations such as edge points, distance maps, differential characteristics,... .

The two steps approach presented in the previous section separates the fitting process into two independent parts. First, a deformation of the shape according to an attraction force, and a parameter recovery from the deformed shape. Consequently, the shape deformation can be done according to a criterion which characterizes the data to be fitted. If we want the hyperquadric to fit edge points we can use the potential originally used in [10]:

$$
P(x, y, z)=-|\nabla \mathcal{I}(x, y, z)|^{2}
$$

characterizing edge points as the minima of the functional $E$ in Eq. (14). We can also consider edge points given by an edge extractor by smoothing these edge points and consider

$\mathrm{RR} \mathrm{n}^{\circ} 2188$ 
the previous potential, or by computing a distance map $d$ which measures at each point the distance to the closest edge point (see [6] for a discussion on these potentials) and take the potential:

$$
P(x, y, z)=d(x, y, z)^{2}
$$

or consider other types of potentials based on this distance map $[5,6]$.

These potentials define different attraction forces. These forces are obtained by the equation:

$$
\vec{f}(x, y, z)=-\nabla P(x, y, z)
$$

We have noted in our experiments that the hyperquadric model performs better if the shape is deformed in the direction of the normal. As expected, the minimization of $E$ for hyperquadric parameters recovery converges in few iterations of the Levenberg-Marquardt method, since the shape is parameterized by a radial numerical parameterization. Thus we apply to the shape only the normal component of the force:

$$
\overrightarrow{f_{n}}(x, y, z)=-(\nabla P(x, y, z) \cdot \vec{n}) \vec{n}
$$

where $\vec{n}$ is the normal to the hyperquadric, it can be obtained analytically from the implicit equation of $H$ defined in Eq. (9) (see [9]) or numerically from the numerical parameterization.

The use of similar potentials in the two steps approach proposed in the previous section permits the definition of different error of fit functions which model several problems encountered in image processing and extend the classical approach dedicated to data fitting.

\subsection{Adding terms: Refining the fit}

In the previous sections we have discussed how to fit an hybrid hyperquadric to data points assuming a fixed number of terms in Eq. (9). We now present another contribution for efficiently adding terms to the hyperquadric equation as they are needed, which means that terms should be added to refine the fitting process. After showing how a hyperquadric is deformed when adding a power or an exponential term, we give a way to choose these terms such that the shape deformation is only local. We then define a criterion for automatically 
refining the fit. This method is based on a geometric approach characterized by the inside set $\Omega$ associated to each hyperquadric. This set is defined by:

$$
\Omega=\left\{(x, y, z) \in \mathbb{R}^{3} \text { s.t. } H(x, y, z) \leq 1\right\},
$$

where $H$ is a hyperquadric defined by Eq. (3), whereas the shape is defined by $\mathcal{S}$ given in Eq. (15).

There are two types of terms that can be added, a power of a 3-D affine form or an exponential term. The exponential term allows to introduce concavities in the hyperquadric shape. In the following the added term will be noted $H_{0}$, and the new hyperquadric is defined by:

$$
H_{1}(x, y, z)=H(x, y, z)+H_{0}(x, y, z)=1 .
$$

Let $\Omega_{0}$ and $\Omega_{1}$ be the set associated as in Eq. 20 with $H_{0}$ and $H_{1}$ respectively. Since $H_{1}(x, y, z) \geq H_{0}(x, y, z)$ and $H_{1}(x, y, z) \geq H(x, y, z)$, the set $\Omega_{1}$ is included in the other ones. Hence we have $\Omega_{1} \subset \Omega_{0} \cap \Omega$ and for $\Omega_{1}$ to be not empty, $\Omega$ and $\Omega_{0}$ have to intersect. At points of $\mathcal{S}$ where $H_{0}$ is close to 0 , the shape is not changed much while where $H_{0}$ is greater it affects the shape of $\mathcal{S}$. To introduce a slight deformation in the shape $\mathcal{S}$, we have to choose $H_{0}$ small enough all over the set $\Omega$ and such its larger values are in the area to be deformed.

\subsubsection{Addition of a power term}

When adding to the hyperquadric a power of a 3-D affine form like

$$
H_{0}(x, y, z)=\left|a_{1} x+a_{2} y+a_{3} z+a_{4}\right|^{\gamma},
$$

the set $\Omega \cap \Omega_{0}$ must not be empty. The set $\Omega_{0}$ is a strip (see Section 2.2) and for each term $H_{0}$ the center area of this strip represents the smallest values of $H_{0}$ and the highest values are on the hyperplanes. This allows to modify the shape $\mathcal{S}$ in a non symmetric manner by choosing the center of the strip $\Omega_{0}$ outside the set $\Omega$. Adding such terms with well chosen parameters allows to blunt a part of $\mathcal{S}$ while keeping it convex.

Han [8] have proposed a method for adding polynomial terms to refine the fitting based on splitting polynomial terms. This split was done on each term and the authors select the $\mathrm{RR} \mathrm{n}^{\circ} 2188$ 
term which has the lower error of fit. This method is computationally expensive and yields generally a higher number of terms than necessary. In the following we present a method based on the error of fit function (14) and a geometric interpretation of the added polynomial term. This last feature characterizes our approach since it allows a geometric control on the convex polytope $\Omega$.

We start the algorithm with a fixed number of terms, once we have attained a minimum of the energy (14) we test the goodness of the fit. If the accuracy of the fit satisfies a given requirement (typically we compare the energy with a given value), we stop the fitting process, otherwise we add a polynomial term. Adding a polynomial term is done through the definition of a function which characterizes the disparities between the hyperquadric surface and the data we are trying to fit. These data are not necessarily the exact data points we are trying to fit, since our algorithm (described in section 4.3) is iterative and hence at each iteration, in the second step, we try to fit the points obtained after applying the force $f$ (Eq. (19)) to the previous hyperquadric surface.

We define

$$
\mathcal{C}=\operatorname{sign}(\vec{n} \cdot \vec{f}) P
$$

over the whole shape $\mathcal{S}$ where $\vec{n}$ is the outward normal and $\vec{f}$ is the attraction force. This function represents a measure of the relative position of the recovered shape and the initial data points. Locally it measures if a data point is inside or outside the recovered shape. This criterion is more general than a classical inside-outside function where the points to be fitted are already known. Its definition, based on the force $\vec{f}$ attracting the hyperquadric toward the data points, measures the relative position between the current hyperquadric and the data points.

If in a given area $\mathcal{C}$ is negative, the recovered shape $\mathcal{S}$ embodies the data points. To refine this fitting we add a power term defined in the following way: we choose the point $X_{d}$ of $\mathcal{S}$ where $\mathcal{C}$ is negative which has a maximal force magnitude. We then consider at this point the affine plane $D_{1}$ (or line in 2-D case) $a_{1} x+a_{2} y+a_{3} z+a_{4}=1$ which is orthogonal to the attraction force $\vec{f}$ at this point. The second algebraic equation $a_{1} x+a_{2} y+a_{3} z+a_{4}=-1$ is chosen such that $\Omega$ is included in the strip $\Omega_{0}$. Since we want to affect the shape of $\Omega$ only 

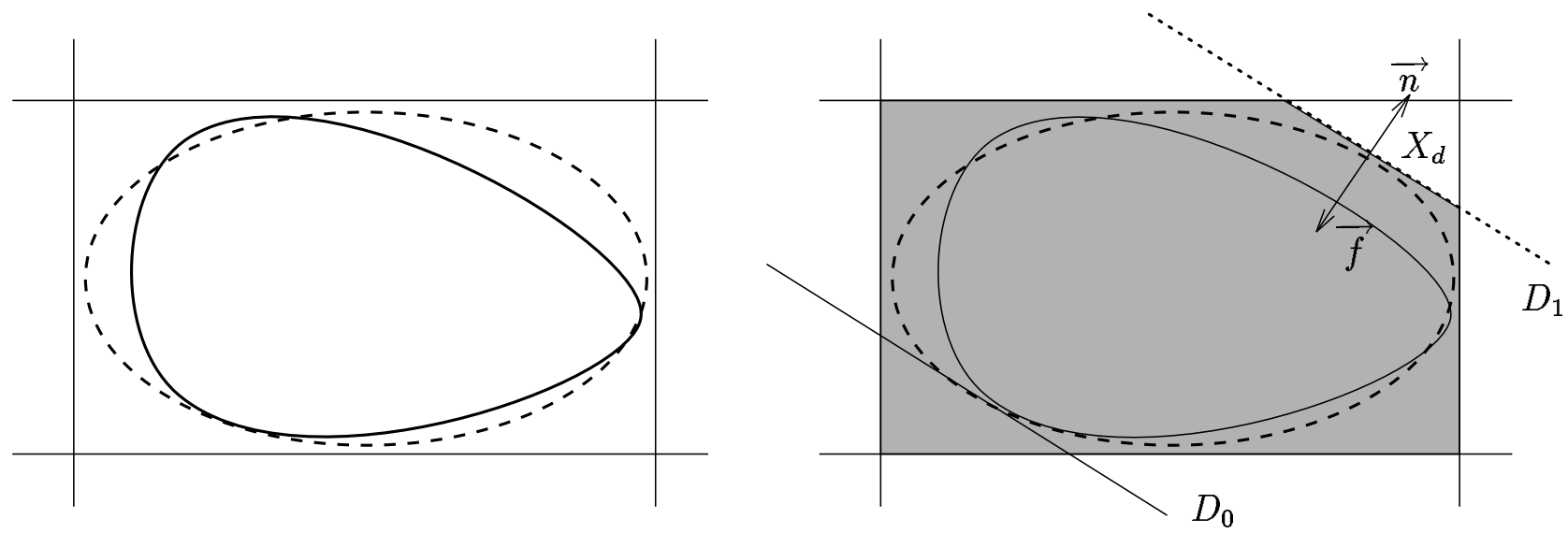

Figure 5: An illustration of the geometric approach for adding a power term to refine the fitting process. On the left we represent a triangular form (in solid lines) fitted by a two terms hyperquadric model (in dashed lines) and the associated strips. The figure on the right shows the definition of the added power term according to the criterion $\mathcal{C}(21), D_{1}$ and $D_{0}$ represent respectively the equations $H_{0}(x, y)=a_{1} x+a_{2} y+a_{3}=1$ and $H_{0}(x, y)=a_{1} x+a_{2} y+a_{3}=0$ associated with the added power term. The convex polytope of the new hyperquadric is drawn in gray.

$\mathrm{RR} \mathrm{n}^{\circ} 2188$ 

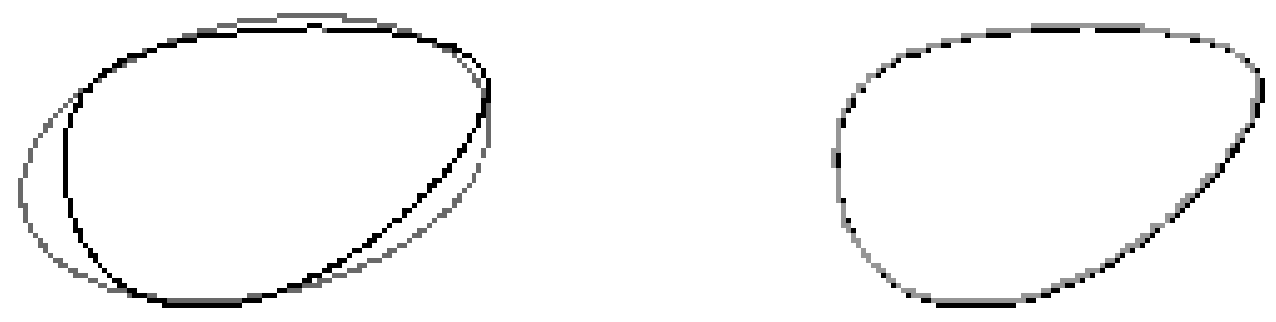

Figure 6: An example of adding polynomial term. In the left we show the fit of a triangular shape (in black) with an hyperquadric defined by two terms (in gray). This triangular shape is accurately recovered by adding a polynomial term with the proposed approach.

locally, we constrain also the strip $\Omega_{0}$ to have the opposite side of $\Omega$ in the area where $H_{0}$ is small. An illustration of the geometric definition of these terms is given in Fig. 5 where we also show the change of the convex polytope associated to the hyperquadric when add a power term.

In figure 6 we illustrate this method, the first figure shows the obtained shape with two polynomial terms, and as expected the two terms are not sufficient for recovering a triangular like shape. The second figure shows the obtained shape once we have added a term by considering the approach explained above. Once we have set the new term, the shape is recovered in few iterations.

In figure 7 we show an example of recovering image boundaries based on hyperquadrics. In this example we process a Magnetic Resonance Image for extracting the contours of a ventricle and an auricle. In this figure the two structures were extracted by using a 6 -terms hyperquadric. 

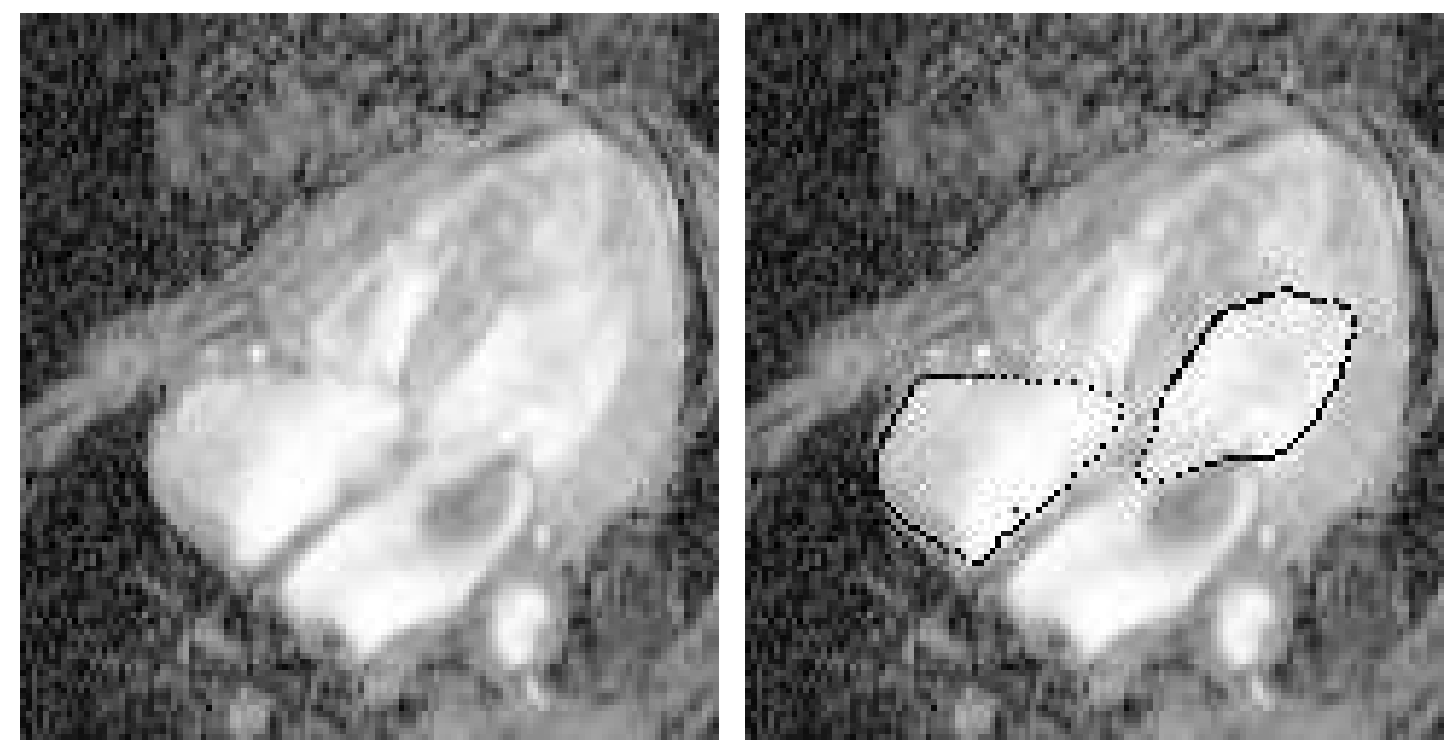

Figure 7: Extracting cavities boundaries in a MRI image of the heart.

The Fig. 8 shows a new type of remote sensing images. This high resolution radiometer image obtained with the NOAA-12 satellite measures the sea surface temperature of the west french coast. In this type of images we are interested in segmenting the vortex appearing in the image they correspond to a mix of cold and heat water-fronts. In figure 8 we show the segmentation of the vortex edge points with a 3-term hyperquadric model.

\subsubsection{Addition of an exponential term}

In this case we consider:

$$
H_{0}(x, y, z)=\mathrm{c} \mathrm{e} \sum^{-\sum_{l=0}^{L}\left|K_{l}(x, y, z)\right|^{\gamma_{l}}}
$$

Adding an exponential term to the hyperquadric allows to introduce a concavity in the shape $\mathcal{S}$ while keeping a smooth shape. The shape of the concavity is completely defined by $\Omega_{0}$ which is the outside of the hyperquadric given by $\sum_{l=0}^{L}\left|K_{l}(x, y, z)\right|^{\gamma_{l}}=\log c$. If $c \leq 1$ then the set $\Omega_{0}$ is the full $3-\mathrm{D}$ space. Since $H_{0}$ has a fast decay in $\Omega_{0}$, it deforms the shape $\mathcal{S}$ only locally to introduce a concavity. In Fig. 9 we have used a 3 terms hyperquadric and added a concavity through an exponential term. In this example the bounding polygon of $\Omega$ defining $\mathrm{RR} \mathrm{n}^{\circ} 2188$ 

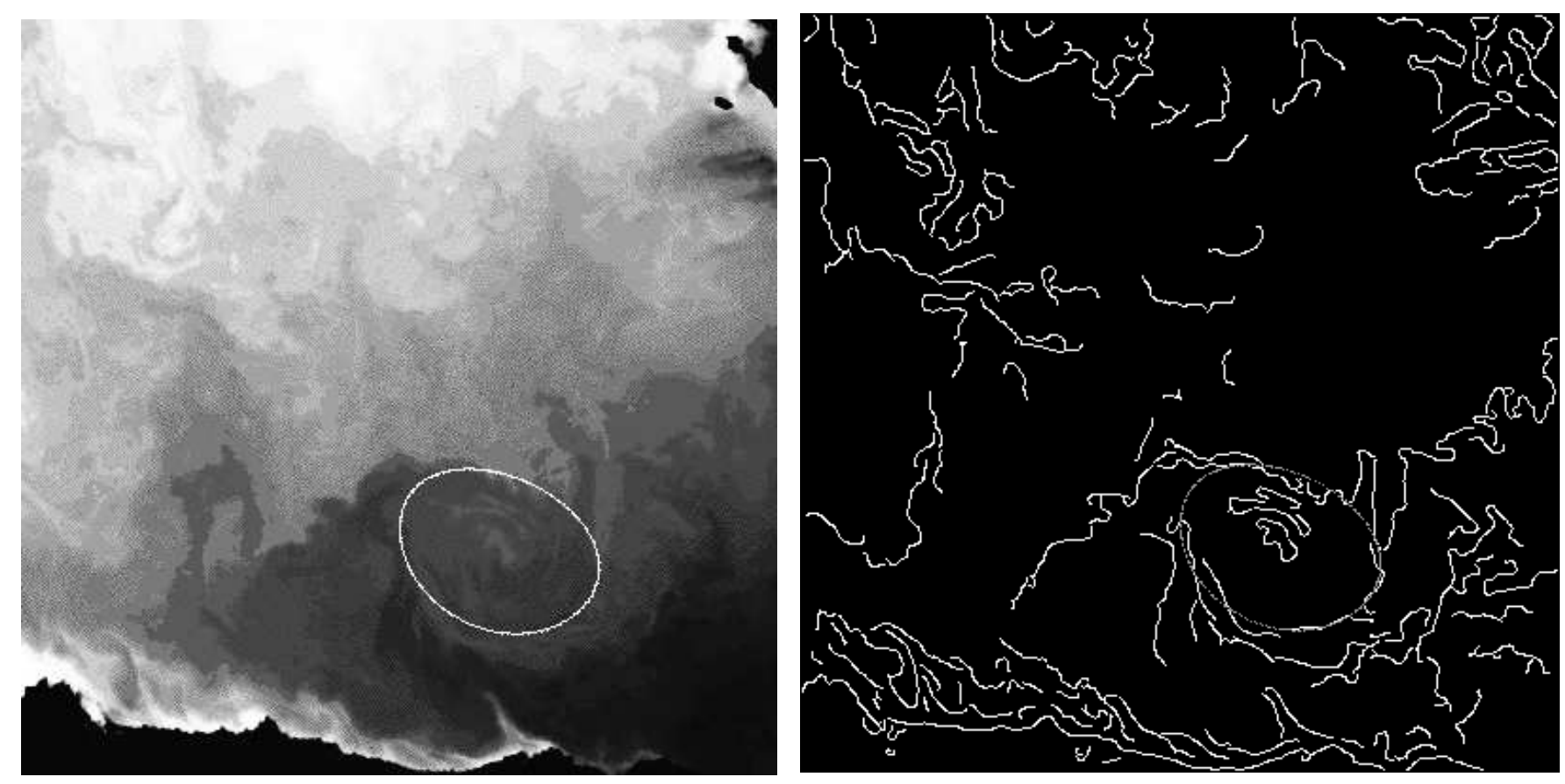

Figure 8: Fit of a 3 terms hyperquadric to the edge points of a vortex in a NOAA-12 surface sea temperature image of the west french coast. We show an overlay of the obtained hyperquadric on the image (where the gray level where enhanced to visualize the vortex) to and its edge points.

the hyperquadric and of $\Omega_{0}$ defining the concavity are drawn in gray. Once we have defined the set $\Omega_{0}$ it defines also the local shape deformation of the initial hyperquadric $H$.

In the fitting process, adding an exponential term to the hyperquadric is based on the same criterion as defined by the function $\mathcal{C}$ of Eq. (21). Once we have detected those points where $\mathcal{C}$ is negative we choose the point $(x, y, z) \in \mathbb{R}^{3}$ such that $|\mathcal{C}|=P$ is maximal and define a concavity centered at this point $X_{c}=\left(x_{c}, y_{c}, z_{c}\right)$ by choosing appropriately the parameters of the exponential term. Indeed, we consider the point $X_{d}=\left(x_{d}, y_{d}, z_{d}\right)$ given by displacing the point $X_{c}$ according to the attraction force. The distance between these two points gives the half width of a first strip centered in $X_{c}$ and oriented by the hyperquadric tangent at the point $X_{c}$. The second strip is also centered at $X_{c}$ and oriented by the hyperquadric normal. Its width is given by searching around $X_{c}$ the points $p_{1}$ and $p_{2}$ minimizing $\mathcal{C}(21)$. These two 

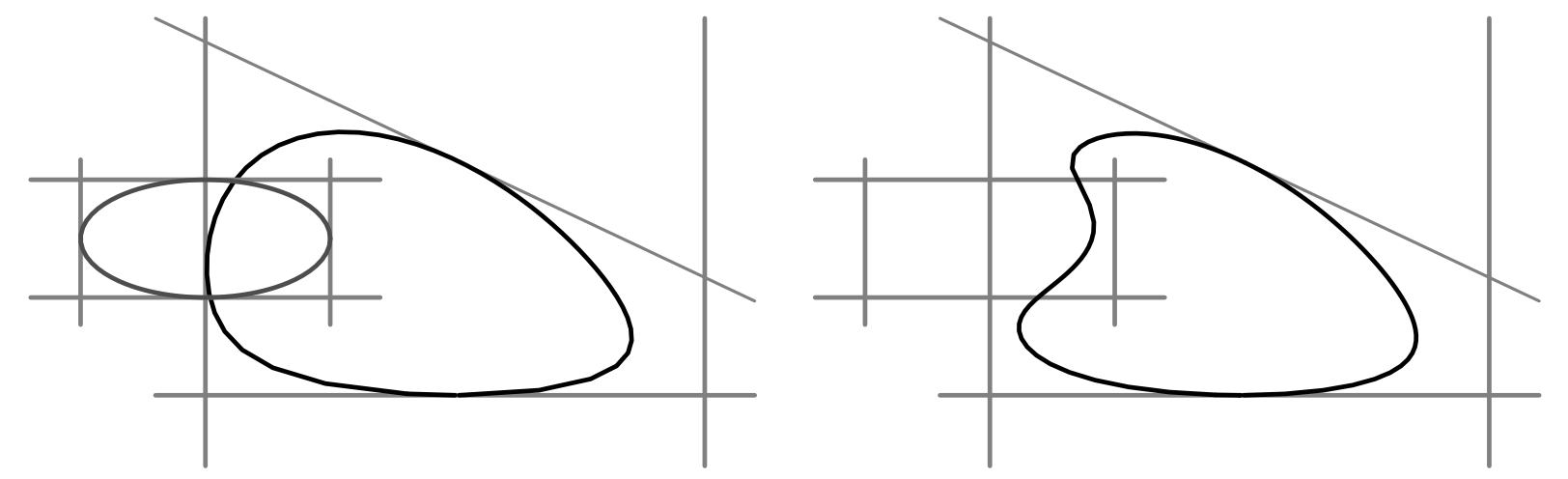

Figure 9: This figure illustrates the geometric definition of a concavity through the definition of its domain. The first figure shows the initial hyperquadric defined by three terms with its bounding polygon and the ellipse defining the concavity. The second figure shows the obtained hybrid hyperquadric shape. The local control of the shape is given by the local definition of the added exponential term. The localization and the size of the ellipse define the local properties of the deformed hyperquadric.

strips define a two terms hyperquadric and consequently the shape of the added concavity. An illustration of the definitions of these strips is given in Fig.10. In this figure the data points are in solid lines and the recovered hyperquadric before adding exponential term is drawn in dashed lines.

The last coefficient $c$ in Eq. (22) can also be estimated from the current hyperquadric $H$ evaluated at the point $X_{d}$. We look for $c$ such that

$$
H_{1}\left(x_{d}, y_{d}, z_{d}\right)=H\left(x_{d}, y_{d}, z_{d}\right)+\mathrm{c} \mathrm{e}^{-\sum_{l=0}^{L}\left|K_{l}\left(x_{d}, y_{d}, z_{d}\right)\right|^{\gamma_{l}}}=1
$$

which yields:

$$
c=\left(1-H\left(x_{d}, y_{d}, z_{d}\right)\right) \mathrm{e}^{\sum_{l=0}^{L}\left|K_{l}\left(x_{d}, y_{d}, z_{d}\right)\right|^{\gamma_{l}}} .
$$

$\mathrm{RR} \mathrm{n}^{\circ} 2188$ 


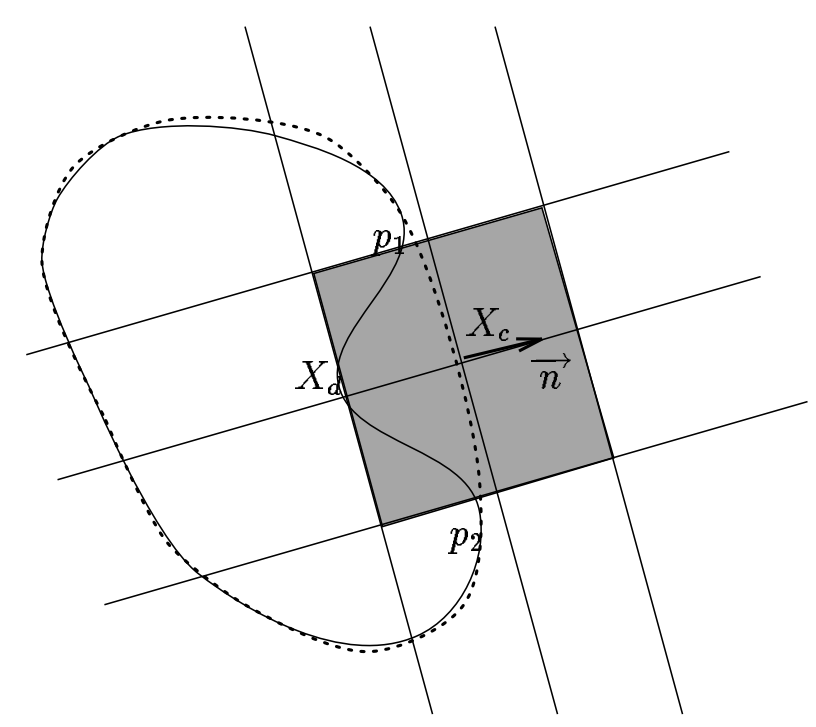

Figure 10: An illustration of the proposed geometric method for adding a concavity in a hyperquadric shape. The shape to be recovered is drawn in solid lines and the fitted hyperquadric is given in dashed lines. The gray area represents the bounding polygon of set $\Omega_{0}$ defining locally the shape and the location of the concavity. This set is defined by the point $X_{c}$ (maximal value of $\mathcal{C}$ ) and $X_{d}$ is obtained by displacing $X_{c}$ according to the force $\vec{f}$. These two points define the first strip, the second strip is defined by the points $p_{1}$ and $p_{2}$ (local minima of $\mathcal{C}$ ) and the outward normal $\vec{n}$.

In the different experiments done with our method, we have found that taking $L=2$ yields a sufficient variety of allowable shapes. Naturally we can increase this number of term by considering a similar approach as described in section 4.5.1.

In figure 11, we tried to fit the data points with a hyperquadric curve (Fig. 11 left). In this example we have started the fit with two terms and we have added an other polynomial term ending with a representation of the shape obtained with three terms, but the obtained fit is not accurate. Alternatively, by introducing two concavities with our method, as described above, we obtain an accurate fit of the data (Fig. 11 right).

In figure 12 we give an example of fitting a hyperquadric surface to 3 -D data points. These points were obtained from a 5 -terms hyperquadric with 3 concavities. Our hybrid 

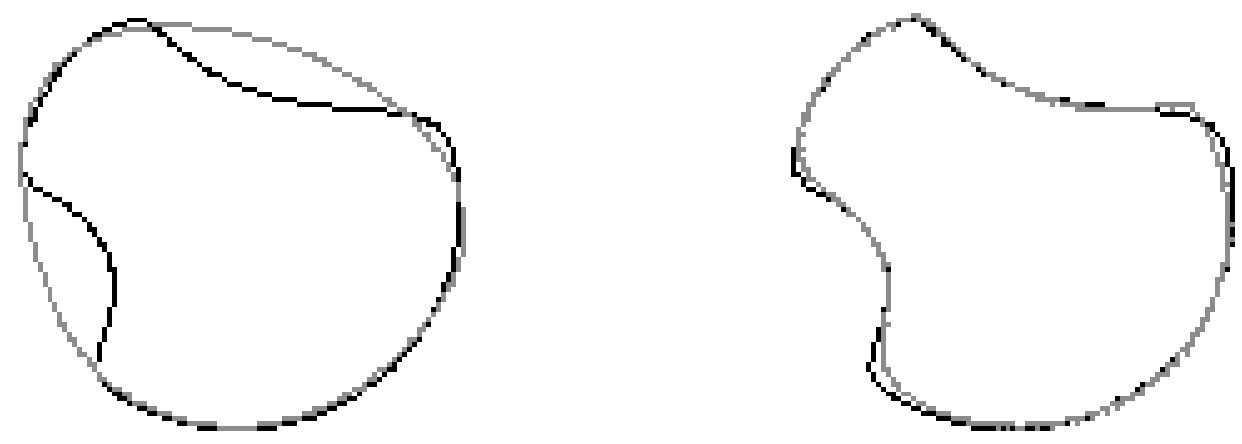

Figure 11: An example of fitting our hybrid model to non convex data points. The first figure (left) shows the obtained three terms hyperquadric. As expected we could not fit accurately the data by adding power terms as described in section 4.5.1, contrarily using a hybrid hyperquadric model yields an accurate fit of the data. In this example we have started with a 2 terms hyperquadric model and added two exponential terms as described in section 4.5.2.

model with the geometric approach of adding polynomial and exponential terms allows to recover accurately the $3-\mathrm{D}$ data points.

In the next section we give the mathematical background, showing clearly the local definition of the model, allowing to deform locally the shape of a given hybrid hyperquadric.

\subsubsection{Justification of the local character of the deformation}

We suppose a couple $\left(X_{0}, r_{0}\right)$ is given where $X_{0}$ is a point on the hyperquadric and $r_{0}$ is the distance to which this point has to be moved. An error $\delta>0$ is admitted for displacement of the hyperquadric points outside a neighborhood of $X_{0}$.

We give here the main idea of the way to choose an extra term being sure that no point on the hyperquadric outside the ball centered at $X_{0}$ of radius $r_{0}$ is moved of more than $\delta$.

$\mathrm{RR} \mathrm{n}^{\circ} 2188$ 


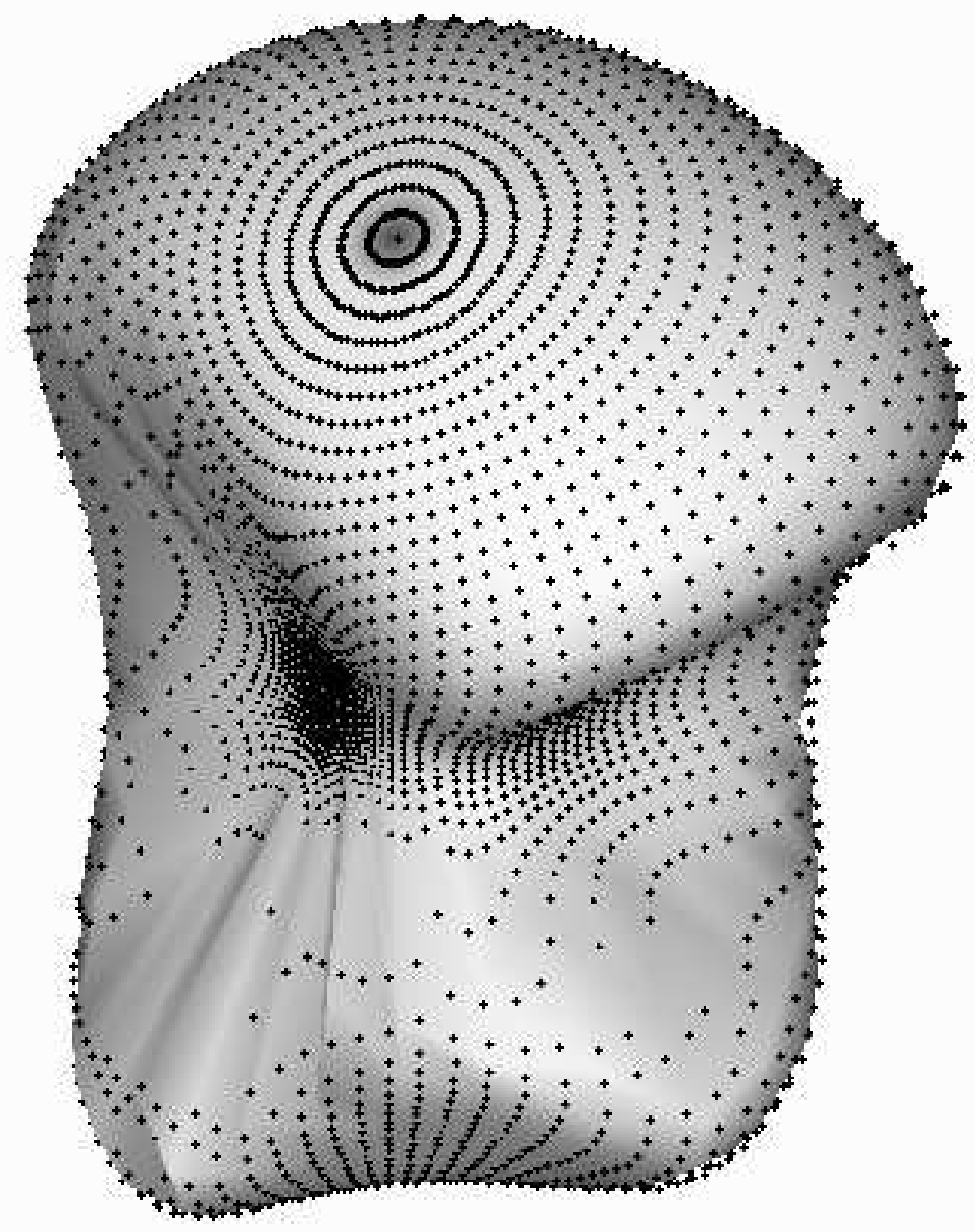

Figure 12: A fit of 3-D data points with a hybrid hyperquadric. The obtained surface is rendered, the dots represent the given 3-D data 
Lemma 1 There is an $\epsilon_{0}>0$ such that if $0<\epsilon<\epsilon_{0}$, when deforming the hyperquadric $H=1$ to the other hyperquadric $H=1-\epsilon$, no point is moved more than $\delta$.

We mean by this that the distance between a point of the new hyperquadric and the first hyperquadric is less than $\delta$. This is due to the continuity of the equation $H=\alpha$ with respect to $\alpha$ using a radial parameterization for example. This can be seen directly using the estimation of the distance (12) of a point $X$ such that $H(X)=1-\epsilon$ and the first hyperquadric $\mathcal{S}$ of equation $H=1$ :

$$
d(X, \mathcal{S}) \simeq \frac{|H(X)-1|}{\|\nabla H(X)\|}=\frac{\epsilon}{\|\nabla H(X)\|}
$$

This shows that assuming there is no singular point on the hyperquadric, points on the new hyperquadric can be made close enough to the first one.

Lemma 2 We can choose $c$ and $K$ such that adding to the hyperquadric equation the term $H_{0}=c e^{-K}$, the deformation is only local on a radius $r_{0}$ around $X_{0}$ to an error within $\delta$.

Using $\epsilon$ given by Lemma 1 , we have to choose $c$ such that $H_{0}<\epsilon$ for $\left\|X-X_{0}\right\|>r_{0}$, For example we can take $K=\left\|X-X_{0}\right\|^{2}$ (in our example, we have in fact two axes and $K$ is elliptic, $K=\left\{\frac{x-x_{0}}{r_{1}}\right\}^{2}+\left\{\frac{y-y_{0}}{r_{2}}\right\}^{2}$. We have $\left\|X-X_{0}\right\|^{2}>r_{0}^{2}$ implies $c e^{-K}<c e^{-r_{0}^{2}}$. It is sufficient to choose $c$ satisfying $c \leq \epsilon e^{r_{0}^{2}}$.

\subsubsection{Adding terms: An algorithm}

In this section we describe the algorithm used to add terms in the implicit representation of the shape. As mentioned before, adding terms is used to refine the fit to obtain an accurate representation of the data. An added term can be of two types: power or exponential term. In the previous sections we have described the geometric definition of the coefficients of the added term, here we summarize the used algorithm.

1. Start the fit with a given number of parameters with a classic hyperquadric,

2. Minimize the energy $E(\mathcal{A})(14)$ until we reach a stationary solution (i.e. until the energy $E(\mathcal{A})$ does not decrease)

$\mathrm{RR} \mathrm{n}^{\circ} 2188$ 
3. Test for the accuracy of the fit (typically if the error of fit is smaller than a given value):

- while the accuracy of the fit is not sufficient, we add a power term according to the method described in section 4.5 .1 and process stage 2 .

- while the accuracy of the fit is not sufficient, we add an exponential term to introduce concavities according to the method described in section 4.5 .2 and process stage 2 .

\section{Hybrid Hyperquadric Fitting From Very Sparse Data}

The hybrid hyperquadric fitting described in the previous sections is based on deforming hyperquadric points according to an attraction force and recovering the hyperquadric parameters by minimizing the functional (11). This second step have to take into account the sparseness of the data since minimizing functional (11) gives the same weight to all data points. Hence if the given data are very sparse we will not recover the shape of the given data. In some other works the authors fit the shape according to the data lying in the normal direction of the initial shape but these approaches assume that the location of the data points is known. This assumption is not valid when using an energy minimization approach like the one proposed in section 4.2. Nevertheless, in this section we propose a new method for defining the weight for each data point according to the sparseness of the data. This method is based on the dot product between the outward normal of the shape and the attraction force derived from the potential $P(18)$. Hence, once we have displaced the hyperquadric points according to the force $\vec{f}(19)$, we recover the hyperquadric parameters by minimizing:

$$
\sum_{d=0}^{D}\left|\frac{\vec{f}}{\|\vec{f}\|} \cdot \vec{n}\right|\left|H\left(x_{d}, y_{d}, z_{d}\right)-1\right|^{2}
$$

where $D$ is the number of points tesselating the shape of the hyperquadric.

This criterion allows to weight data points according to the attraction force and the hyperquadric normal. Points lying in the normal direction will be weighted to one while 


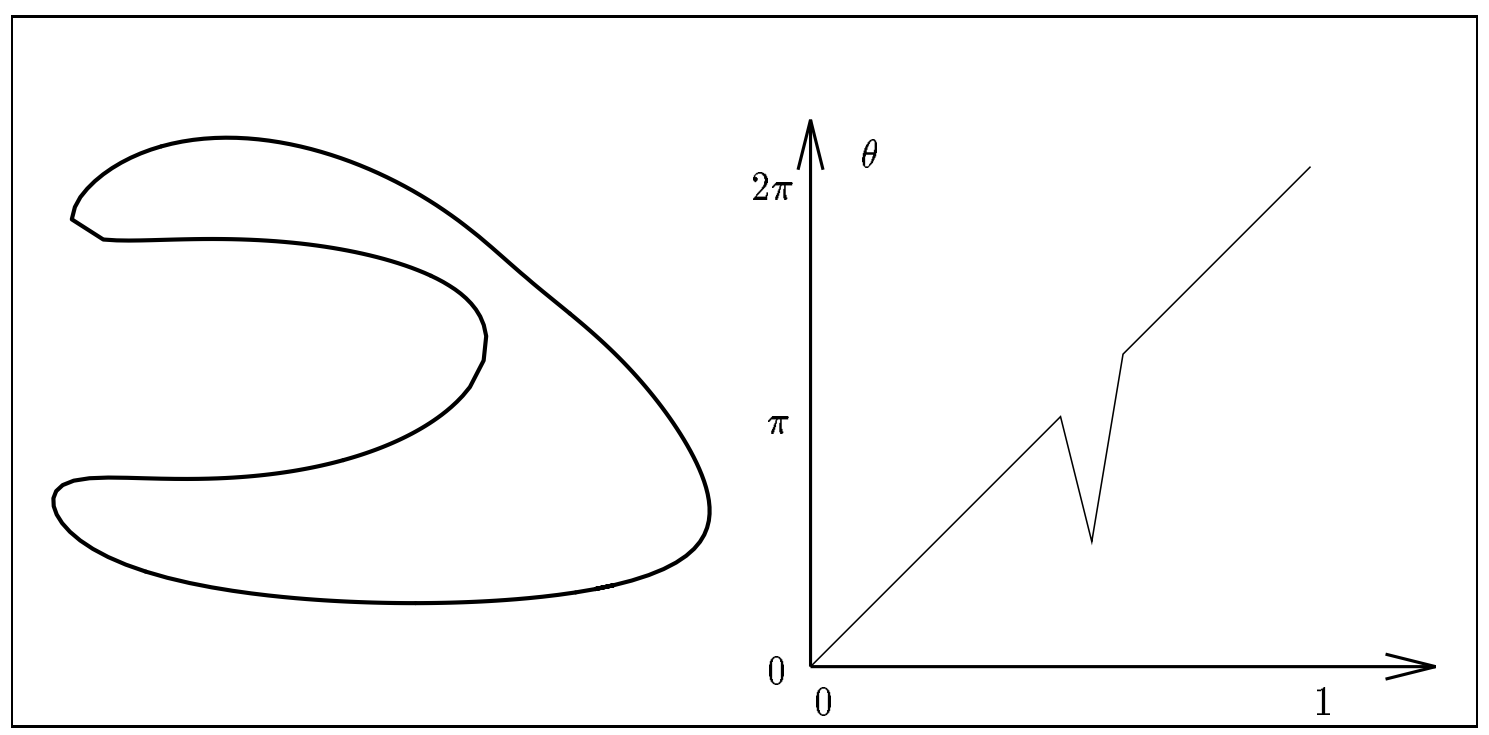

Figure 13: A numerical parameterization of a 2-D hybrid hyperquadric, where the angle $\theta \in[0,2 \pi[$ is no longer monotonous. In the left we represent a hybrid hyperquadric defined by three polynomial term and a concavity term, on the right we represent the function $\theta \in[0,2 \pi[$ allowing to describe efficiently the shape of the parametric model.

points lying in other directions will be weighted according to the dot product between the attraction force and the normal direction. This weighting function allows to measure the sparseness of the data with regard to the numerical tessellation of the surface.

\section{Generalization of the Numerical Radial Paramete- rization}

The use of a numerical radial parameterization gives an efficient description of the shape. This description does not constrain the set of allowable shapes in the case of hyperquadrics, since the recovered shape is convex (for $\gamma_{i} \geq 1$ ). When we consider hybrid hyperquadrics shapes, the use of a classical radial parameterization constrains the set of allowable shapes. Indeed the radial parameterization can only describe a curve (or surface) that is star-shaped with respect to the center point of the parameterization. This means that for all point $M \in$ $\mathcal{S}_{\mathcal{A}}$ on the hybrid hyperquadric, the line segment joining the center of the parameterization

$\mathrm{RR} \mathrm{n}^{\circ} 2188$ 
to $M$ lies entirely within the hyperquadric (in $\Omega$ ) [13]. Hence the radial parameterization cannot always describe a hybrid hyperquadric shape since all the points satisfying Eq. (9) cannot be "seen" from the center point of the parameterization.

A way to overcome this constraint, is to consider a multivalued radial parameterization of the parameters $\theta$ and $\varphi$. For each $(\theta, \varphi)$ we search the different scalars $r$ minimizing

$$
|H(X(r, \theta, \varphi))-1|^{2}
$$

where $X=(x, y, z)$ like in section 2.3. But instead of stopping after the first solution we go on until the above expression remains monotonous. This corresponds to reach the bounding polygon of the hyperquadric. Thus the angle $\theta$ (respectively $\varphi$ ) is no longer a monotonous function scanning the interval $[0,2 \pi[$ (resp. $[-\pi / 2, \pi / 2[$ ) but depends on the shape of the hybrid hyperquadric. This method amounts to have a multivalued function defining the radius of the numerical parameterization. A second step consists in sorting this set of points to describe the hyperquadric as a parameterized curve (or surface). We give an illustration of such a technique in figure 13 where the variable $\theta \in[0,2 \pi[$ spans this interval according to the parameters of the hybrid hyperquadric. The obtained shape cannot be represented with a classical radial parameterization since it is not star-shaped.

This new numerical parameterization can be characterized through the definition of a function

$$
(u, v) \in[0,1] \times[0,1] \longmapsto(r(u, v), \theta(u, v), \varphi(u, v)) \in \mathbb{R}^{3}
$$

where $(u, v)$ parameterizes the set of points $X=(x, y, z)$ satisfying equation (9). Defining such a function allows to describe the set of points satisfying the hybrid hyperquadric equation (9) according to different criteria such as arc length, and consequently to incorporate the characterization of the solutions of equation (9) and the sorting of these points in the same process.

In figure 14 we give some complex 2-D shape that can be obtained with this new numerical parameterization. This method can handle non connected shapes. This method applies also for 3-D hybrid hyperquadric, in this case the surfaces are represented through a Delaunay triangulation of the set of points satisfying Eq. (9). An example of non star shaped hybrid hyperquadric surface is given in figure 15 . In this figure we show only the isoparametric curves 


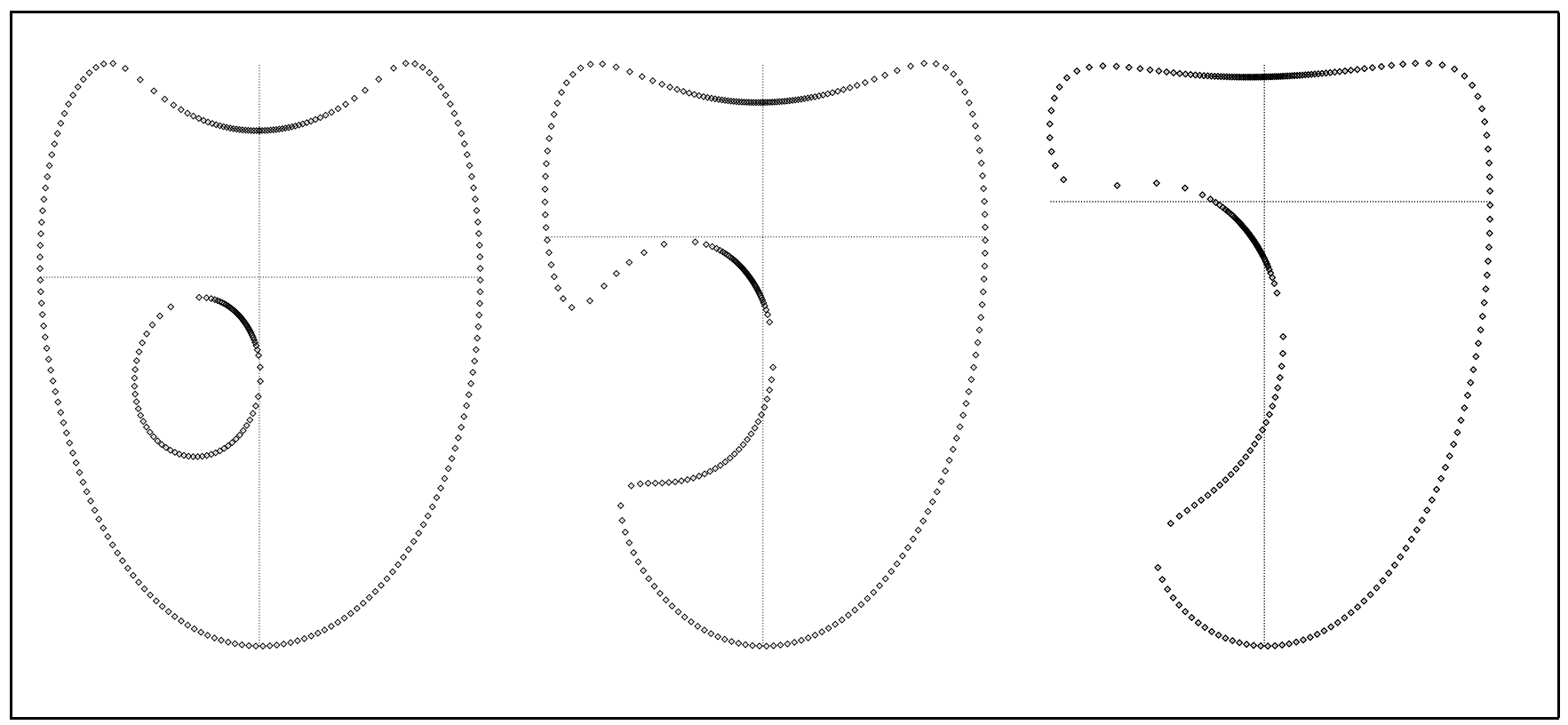

Figure 14: Some examples of the shapes that can be obtained with the new numerical parameterization. This new method allows the description of non star shaped curves and also non connected curves. Remark that the density of points depends on the angle between the curve tangent and the line linking a point to the center of the parameterization (in this example the origin)

defining the surface, this illustrates the fact that adding an exponential in a hyperquadric implicit description can generate non connected shapes which are efficiently described with the new multivalued parameterization.

\section{Conclusion}

We have developed a hybrid hyperquadric model with global and local properties inherited from hyperquadrics. This model allows a wide variety of shape modelling including nonconvex smooth shapes and is henceforth useful for reconstructing $2-\mathrm{D}$ and $3-\mathrm{D}$ shapes. A numerical parameterization of the shape and an energy minimization scheme is proposed for parameters recovery, allowing an accurate fit of the data independently of the number of the

$\mathrm{RR} \mathrm{n}^{\circ} 2188$ 

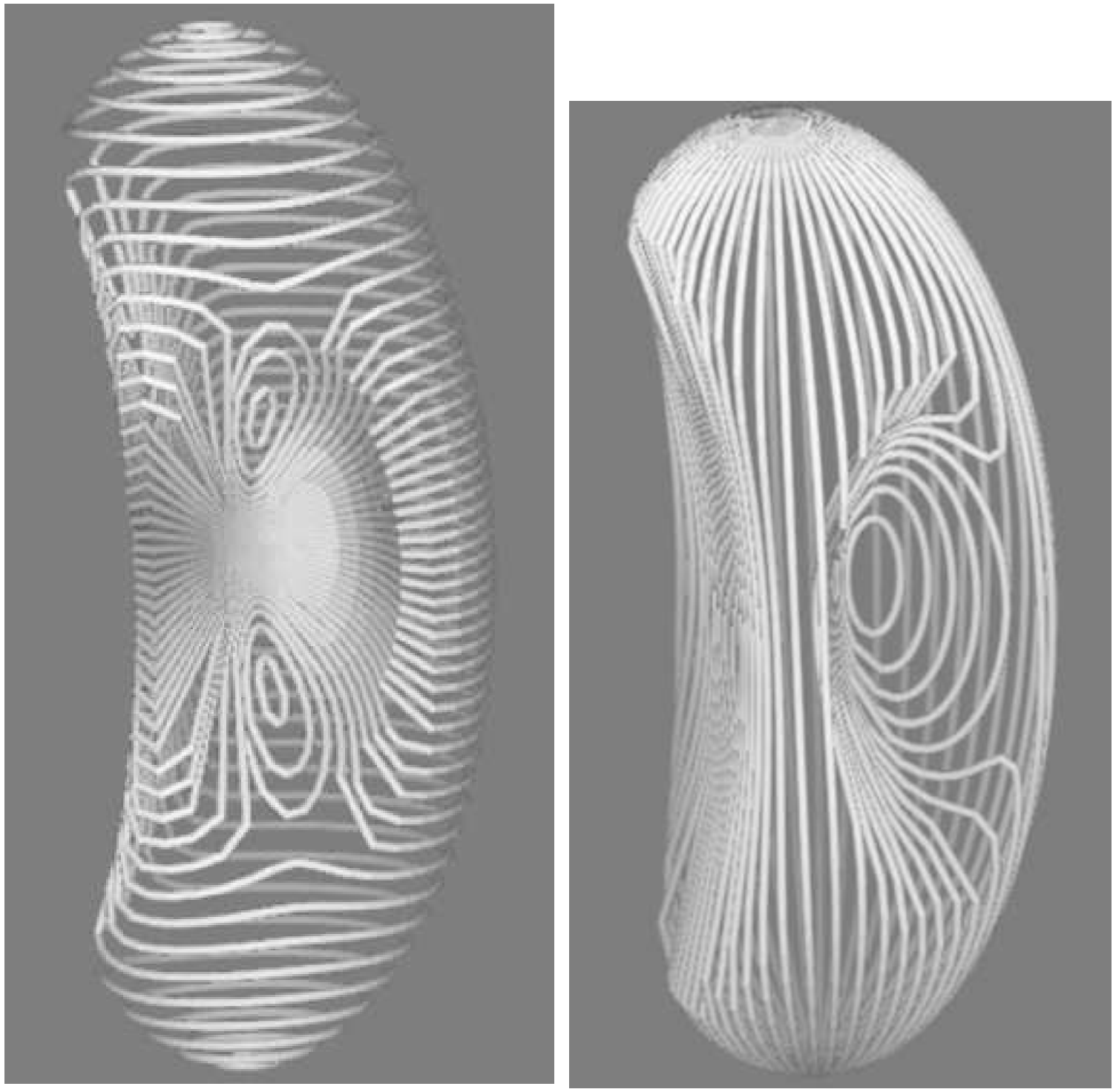

Figure 15: An example of a 3-D non star shaped hybrid hyperquadric surface obtained with the new multivalued parameterization. In this figure we show the plot of the surface through its isoparametric curves illustrating more efficiently its complex shape 
parameters describing the shape. A new geometric approach allows to increase the number of parameters and to automatically add shape concavities to refine the fit. We have also presented a new numerical parameterization to handle non connected shapes and non star shaped objects improving the solution of the hybrid hyperquadric equation.

The obtained parametric model describes global and local properties through a unique implicit equation yielding a representation of the shape by means of its parameters. Further work should be done in the use of the hybrid hyperquadrics parameters for object recognition and for characterizing object deformation from its parameters.

\section{References}

[1] A. H. Barr. Superquadrics and angle-preserving transformations. IEEE Computer Graphics Application, 1:11-23, 1981.

[2] A. H. Barr. Global and local deformations of solid primitives. Computer Graphics, 18(3):21-30, July 1984.

[3] J. F. Blinn. A generalization of algebraic surfaces drawing. ACM Transactions on Graphics, 1(3):235-256, July 1982.

[4] T. E. Boult and A. D. Gross. Recovery of superquadrics from 3-D informations. In Proc. Spatial Reasoning and Multi-Sensor Fusion Workshop, pages 128-137, St-Charles IL, 1987.

[5] Isaac Cohen, Laurent D. Cohen, and Nicholas Ayache. Using deformable surfaces to segment 3D images and infer differential structures. Computer Vision, Graphics, and Image Processing: Image Understanding, 56(2):242-263, 1992.

[6] Laurent D. Cohen and Isaac Cohen. Finite element methods for active contour models and balloons from 2-D to 3-D. IEEE Transactions on Pattern Analysis and Machine Intelligence, 15(11):1131-1147, November 1993.

[7] Philip E. Gill, Walter Murray, and Margaret H. Wright. Practical Optimization. Academic Press, London, 1981.

[8] Song Han, Dmitry B. Goldgof, and Kevin W. Bowyer. Using hyperquadrics for shape recovery from range data. In IEEE Proceedings of the Third International Conference on Computer Vision, pages 492-496, Berlin, June 1993. IEEE.

[9] Andrew J. Hanson. Hyperquadrics: Smoothly deformable shapes with convex polyhedral bounds. Computer Vision, Graphics, and Image Processing, 44:191-210, 1988.

[10] Michael Kass, Andrew Witkin, and Demetri Terzopoulos. Snakes: Active contour models. International Journal of Computer Vision, 1:321-331, 1987.

[11] D. Kriegman and J. Ponce. On recognizing and positioning curved 3-D objects from image contours. IEEE Transactions on Pattern Analysis and Machine Intelligence, 12(12):1127-1137, December 1990.

$\mathrm{RR} \mathrm{n}^{\circ} 2188$ 
[12] A.P. Pentland. Recognition by parts. In IEEE Proceedings of the First International Conference on Computer Vision, pages 612-620, 1987.

[13] Franco P. Preparata and Michael I. Shamos. Computational Geometry, An Introduction. Springer Verlag, 1985.

[14] F. Solina and R. Bajcsy. Recovery of parametric models from range images: The case of superquadrics with global deformations. IEEE Transactions on Pattern Analysis and Machine Intelligence, 12(2):131-147, February 1990.

[15] G. Taubin. An improved algorithm for algebraic curve and surface fitting. In IEEE Proceedings of the Third International Conference on Computer Vision, pages 658-665, Berlin, June 1993. IEEE.

[16] G. Taubin and F. Cukierman. Parameterizing and fitting bounded algebraic curves and surfaces. In IEEE Proceedings of Computer Vision and Pattern Recognition, pages 103-108, Champaign, Illinois, June 1992. IEEE.

[17] D. Terzopoulos and D. Metaxas. Dynamic 3-D models with local and global deformations: Deformable superquadrics. IEEE Transactions on Pattern Analysis and Machine Intelligence, 13(7):703-714, 1991.

[18] Demetri Terzopoulos. The computation of visible-surface representations. IEEE Transactions on Pattern Analysis and Machine Intelligence, PAMI-10(4):417-438, July 1988.

[19] Alan L. Yuille, Peter W. Hallinan, and David S. Cohen. Feature extraction from faces using deformable templates. International Journal of Computer Vision, 8(3), September 1992. 
Unité de recherche Inria Lorraine, Technopôle de Nancy-Brabois, Campus scientifique, 615 rue du Jardin Botanique, BP 101, 54600 Villers Lès Nancy

Unité de recherche Inria Rennes, Irisa, Campus universitaire de Beaulieu, 35042 Rennes Cedex Unité de recherche Inria Rhône-Alpes, 46 avenue Félix Viallet, 38031 Grenoble Cedex 1

Unité de recherche Inria Rocquencourt, Domaine de Voluceau, Rocquencourt, BP 105, 78153 Le Chesnay Cedex

Unité de recherche Inria Sophia-Antipolis, 2004 route des Lucioles, BP 93, 06902 Sophia-Antipolis Cedex

\section{Éditeur}

Inria, Domaine de Voluceau, Rocquencourt, BP 105, 78153 Le Chesnay Cedex (France)

ISSN 0249-6399 